\title{
Une anticipation pédagogique
}

Une expérimentation dans le Land du Brandebourg

Joachim Wilke et Ursula Wilke

\section{CpenEdition}

\section{Journals}

Édition électronique

URL : http://journals.openedition.org/ries/4176

DOI : $10.4000 /$ ries. 4176

ISSN : 2261-4265

Éditeur

Centre international d'études pédagogiques

Édition imprimée

Date de publication : 5 mars 1995

Pagination : 143-148

ISSN : $1254-4590$

Référence électronique

Joachim Wilke et Ursula Wilke, "Une anticipation pédagogique », Revue internationale d'éducation de Sèvres [En ligne], 05 | 1995, mis en ligne le 16 mars 2015, consulté le 02 mai 2019. URL : http:// journals.openedition.org/ries/4176 ; DOI : 10.4000/ries.4176

Ce document a été généré automatiquement le 2 mai 2019.

(c) Tous droits réservés 


\title{
Une anticipation pédagogique
}

\author{
Une expérimentation dans le Land du Brandebourg
}

\author{
Joachim Wilke et Ursula Wilke
}

1 Lors de la dernière rentrée scolaire, l'expérience la plus importante entreprise récemment dans le domaine éducatif en Allemagne est entrée dans sa phase ultime. Cette expérience a été réalisée dans le Land du Brandebourg où 44 écoles, depuis deux ans déjà, ont mis à l'épreuve les tenants et les aboutissants d'une nouvelle matière d'enseignement, baptisée L.E.R., c'est-à-dire : Lebensgestaltung-Ethik-Religion. Le premier terme, difficile à traduire, concerne la façon personnelle de former sa manière de vivre au sein d'un contexte communicatif, c'est-à-dire dans la vie quotidienne de la classe scolaire. Dans ce sens précis, on pourrait se risquer à parler de "réflexion sur la conduite de la vie » (traduction littérale de l'expression allemande). Les termes suivants n'ont guère besoin de traduction. Mais regroupés, la « réflexion sur la conduite de la vie», l'éthique et la religion annoncent une discipline traversée de tensions, voire de conflits. D'où vient que sa mise en place ait exigé ces trois années d'expérimentation dont le texte présent veut donner un aperçu, en l'accompagnant de quelques rappels historiques.

\section{Une innovation de type " démocratie de base »}

2 Le Brandebourg fait partie des Neue Bundesländer, c'est-à-dire des régions de l'ex-RDA qui, s'associant à la RFA, ont accédé au statut de Land fédéral. Ce statut comporte une sorte d'autonomie culturelle, notamment quant au découpage de l'enseignement scolaire. Or, à la différence des autres Neue Länder, le Brandebourg fut gouverné, dès les élections de 1990, par une coalition des sociaux-démocrates, des verts et des libéraux. Les conditions politiques propres à la région favorisèrent donc la mise en œuvre d'un projet qui date de la flambée démocratique de 1989. Durant les journées de novembre, au moment de la chute du mur de Berlin, l'une des innombrables tables rondes élabora l'idée d'un enseignement nouveau qui devrait ouvrir l'école aux questions, aux problèmes et aux défis de la vie contemporaine. Participaient à cette table ronde des philosophes et des scientifiques progressistes, des pasteurs et des curés représentant la Kirche von unten (l'Église de base), des parents, des élèves, des enseignants. L'une des réunions regroupa 
un millier de personnes. Au terme d'un long débat, un groupe mixte parvint à formuler les éléments de ce qui paraissait réalisable: instituer une nouvelle branche d'enseignement qui combinerait l'approche des problèmes pratiques de socialisation, l'interrogation sur le sens des actions humaines (à partir de l'activité personnelle) et, enfin, l'acculturation philosophico-éthique.

Né à Berlin, issu d'un compromis, ce schéma fut repris dans la région environnante, le Brandebourg. Étant donné le caractère innovant du projet, il fallait vérifier sa viabilité. Aussi le ministère régional de l'instruction, de la Jeunesse et du sport chargea-t-il l'Institut pédagogique du Brandebourg (PLIB) d'effectuer une expérience-modèle. Deux ans furent nécessaires au PLIB pour préciser les programmes d'enseignement, commencer la formation des enseignants et choisir les écoles appelées à servir de bancs d'essai. Ce choix passait par l'accord non seulement des directeurs d'établissement, mais aussi des parents et, avant tout, des élèves.

Le propre de cette nouvelle discipline, selon les termes mêmes du ministère, consiste à axer l'enseignement sur « l'élève avec ses sensibilités, ses problèmes et ses conflits, avec son besoin d'orientation et de décision, avec son désir d'avoir son mot à dire quant à ses conditions de vie tant présentes que futures ». Pour en arriver là, le système des notes ou des points fut suspendu : il n'y a pas de concours d'éthique. Au lieu de cela, l'enseignant et la classe peuvent manifester leur estime devant un bon travail d'élève (essai, protocole, présentation d'un thème). Il s'agit de créer un climat de confiance mutuelle, dépourvu d'angoisse (vertrauensvoll und angstfrei), où les élèves peuvent développer leur créativité propre, tout en s'habituant à tolérer des opinions différentes et à soutenir des conflits. Cet objectif l'emporte sur l'instruction stricto sensu, ce qui n'empêche pas que les enseignants doivent disposer d'un savoir complexe empruntant à la fois à l'histoire, à la sociologie, à l'écologie, à la psychologie, aux arts et aux médias, à l'éthique et aux religions (au pluriel). À cela s'ajoutent le cadre juridique constitué par la Constitution du Land du Brandebourg, la Loi fondamentale de la RFA (Grundgesetz) et les documents de l'ONU relatifs aux droits de l'homme et aux droits de l'enfant. Ainsi, pour les enseignants, la formation spécialisée s'étend-elle sur plus de trois années, et elle vient s'ajouter à leurs charges quotidiennes. Mais le groupe des 132 participants à cette expérience-modèle est hautement motivé. Depuis l'automne de 1992, ils dispensent le nouvel enseignement au niveau du premier cycle du secondaire (Sekundarstufe I, c'est-à-dire les classes 7 à $10 \mathrm{du}$ cursus scolaire allemand, où le primaire commence avec la classe 1 , la terminale étant la $13^{\mathrm{e}}$ ). À ce jour, 44 écoles réparties sur l'ensemble du Land du Brandebourg sont concernées, ce qui fait 7000 élèves. Ces chiffres attestent l'importance de l'expérience, la plus large qui soit en cours actuellement dans le système scolaire allemand.

\section{Comment aborder l'éthique?}

5 Insistons sur ces chiffres : les 7000 élèves sont, en quelque sorte, des volontaires. Les deux heures de la L.E.R. s'ajoutent pour eux à l'emploi du temps régulier de leur niveau scolaire. Cet enseignement constitue donc une charge supplémentaire qu'ils ont acceptée d'assumer, à travers leur participation à la Schulkonferenz (assemblée réunissant les élus du corps enseignant, des parents et des élèves d'une école). Pour quels motifs l'ont-ils acceptée ? Parce que, au vu de l'information préliminaire, ils ont jugé que cela valait la peine, que cela pouvait être quelque chose de bon ou, pour ainsi dire, un bien. Ils l'ont accepté aussi parce que tout n'y était pas prédéterminé, les plans de la L.E.R. n'étant que 
des plans-cadres : bien que définissant certains Lernfelder (champs d'études), ils laissent aux participants, élèves y compris, la liberté de structurer chaque Lernfeld par le choix des sous-thèmes traités et de la façon de les traiter. Sont ici déjà engagées un certain nombre de notions éthiques : la valeur, le bien, le bon et le mauvais, la liberté, l'activité.

On pourrait bien sûr envisager un enseignement de l'éthique qui vise à la présenter : une discipline philosophique qui théorise la morale, celle-ci étant comprise comme système régulateur des conduites libres, c'est-à-dire qui ne sont pas déterminées par la loi juridique, la norme technologique, la contrainte économique, etc. On pourrait encore définir la tâche de l'éthique: dégager un bien suprême, un réseau de valeurs fondamentales, un tissu de normes adéquates, afin d'orienter et de stimuler la créativité morale, offrir des repères à la conscience (Gewissen), instance du jugement moral. Nous pourrions disserter enfin sur l'interpénétration de l'éthique et de la morale, voire sur la place prépondérante à laquelle l'éthique doit prétendre au sein de la philosophie.

Mais à quoi bon ce type de discours aux niveaux de scolarité qui sont ici concernés, les classes 7 à 10 ? La L.E.R. mise sur le vécu éthique et moral qui se traduit dans un vouloirapprendre, un vouloir-faire la découverte de soi-même et des autres, un vouloir-vivre l'expérience des relations complexes et conflictuelles qui se tissent au quotidien de la classe. Il s'agit de prendre conscience de ce qui se joue dans le quotidien scolaire et, à partir de là, de développer le savoir et le jugement moral. Ainsi se poseront les fondements d'une réflexion proprement éthique (au sens philosophique) qui fera partie des matières $d u$ deuxième cycle $d u$ secondaire (Sekundarstufe II). Par ailleurs, l'expérimentation en cours sert à préparer une expansion de la L.E.R. vers le primaire.

8 Actuellement, cet enseignement débute au milieu du cursus scolaire. Il répond là à un besoin évident. Les élèves des classes 7 à 10 se trouvent dans une phase hautement sensible de leur développement individuel et social: puberté, orientation scolaire, orientation professionnelle dans une région frappée par le chômage. Leur vouloirapprendre correspond à un désir de conseil mutuel. D'où la préférence qu'ils donnent à l'aspect de « réflexion sur la conduite de la vie ». Parmi les sous-thèmes, celui auquel va leur préférence s'intitule : « amitié-amour-sexualité ». Aux enseignants d'accompagner le travail de la classe de façon telle qu'il permette d'affronter des problèmes aigus et d'approfondir le jugement éthico-moral.

9 Pendant la durée de la Sekundarstufe I, ce travail s'applique aux Lernfelder : « Les humains comme individus ", "Les humains dans la communauté", "Des menaces et surcharges qui pèsent sur la vie humaine ", "À la recherche d'une vie remplie de sens", "Les humains et leurs religions, conception du monde et cultures », «La formation de la vie personnelle et les perspectives planétaires ». L'élaboration utilise des méthodes très diverses. Ainsi, pour le premier Lernfeld, les élèves peuvent se caractériser eux-mêmes, soit sous la forme d'un rapport oral ou écrit, soit en se dessinant ou en donnant d'euxmêmes une illustration symbolique. Dans le cadre du deuxième champ thématique, on peut jouer au théâtre, présenter des sketches, mais aussi faire des analyses, noter des relations observées dans la classe ou dans l'environnement social.

10 Pour le champ de l'inter-culturalité, on mise sur l'authenticité : inviter ou visiter les représentants de tel groupe, les témoins de tel événement (certaines classes peuvent rencontrer un survivant de la Shoah).

11 La période d'essai a déjà permis de conclure à la viabilité de la L.E.R. Pour l'instruction publique du Brandebourg, cette période devrait aboutir à une généralisation de la L.E.R. 
Tout est prêt pour cela. Mais il reste un problème qui attend toujours une réponse : est-il permis aux ressortissants du Land du Brandebourg d'enseigner la L.E.R. ?

\section{À l'encontre de la tradition dominante}

12 Le problème est issu de certaines particularités de la tradition scolaire allemande. S'il y avait et s'il y a toujours une diversité marquée des conditions régionales, les États allemands partagent aussi un même héritage historique: pendant très longtemps l'enseignement éthico-moral fut dispensé dans le cadre de l'instruction confessionnelle (Religionsunterricht). Celle-ci était du ressort de l'Église, catholique ou protestante selon l'environnement confessionnel, mais elle faisait partie intégrante de l'éducation publique, y compris dans ses aspects financiers. Cette situation, qui provoqua la révolte nietzschéenne, dura des débuts de l'instruction publique (XVIII siècle) jusqu'aux temps de la République de Weimar (1919-1933). Cette période de l'histoire allemande englobe le Kulturkampf, dans lequel le parti social-démocrate et d'autres forces laïques, se réclamant de la loi Ferry et Bert de 1882, réclamèrent la suppression du Religionsunterricht dans le cadre de l'école. Le Kulturkampf fut réglé par un compromis: les parents pouvaient demander que leurs enfants soient dispensés de suivre ce cours et certaines écoles laïques pouvaient de leur côté offrir un enseignement de Lebenskunde, précurseur de la Lebensgestaltung. Cette dernière possibilité ne fut accordée que dans les États de Prusse, de Saxe, de Thuringe et de Brunswick, c'est-à-dire au nord de l'Allemagne. Pour l'époque du nazisme, une information complète fait défaut, mais il va de soi que l'endoctrinement fasciste l'emporta sur tout enseignement éthico-moral.

Après 1945, le cours de religion (Religionsunterricht) fut réintroduit dans les établissements secondaires. En RDA, la reprise fut de courte durée ; l'école prit un caractère décidément laïque, voire athée, écartant tout enseignement religieux. Les paroisses, regroupant les enfants des croyants, leur enseignaient la doctrine chrétienne, tandis que l'école s'occupait de l'éducation civique. Il n'y avait pas à proprement parler d'enseignement éthique.

La RFA restaura la pratique traditionnelle. La Loi fondamentale stipula dans son $7^{\mathrm{e}}$ article que le Religionsunterricht faisait partie de l'instruction publique, sauf dans les rares écoles laïques. Le Religionsunterricht était obligatoire pour chaque adhérent à une confession déterminée, écoliers y compris ; ici aussi, la possibilité restait ouverte pour les parents de "déclarer le départ» de leurs enfants. Les Länder de Brême et de Berlin-Ouest firent exception à la règle, le premier pratiquant un enseignement chrétien supra-confessionnel d'histoire biblique (qui fut consacré par l'article 141 de la Loi fondamentale de la RFA), le second n'offrant l'enseignement confessionnel qu'à titre facultatif. La Loi fondamentale ne s'appliquait pas en effet à la partie ouest de Berlin, placée sous l'autorité des Alliés. Pendant plus de deux décennies, l'éthique ne figure pas parmi les disciplines enseignées dans les établissements scolaires, même si, dès 1946, la Constitution régionale de la Bavière en avait prévu la possibilité formelle. Mais la secousse de 1968 entraîna une marée des "déclarations de départ ", de sorte qu'une partie de plus en plus grande des écoliers pouvait jouer au football ou prendre l'air, pendant que l'autre partie devait s'intéresser non pas tellement au phénomène religieux qu'aux croyances et aux coutumes de l'un ou l'autre culte. Face à la diminution rapide de la seconde catégorie, les Églises catholique et protestante firent pression pour que fût trouvée une solution. 
Ainsi la possibilité formelle d'apprendre l'éthique fut-elle transformée en une réalité obligatoire pour les réfractaires à l'enseignement confessionnel. De là vient la situation qui prévaut aujourd'hui dans la quasi-totalité des Länder allemands: les écoliers et les élèves ont le choix entre un cours de religion et un cours d'éthique obligatoire. L'éthique se trouve par là dans une position subalterne. Elle est considérée comme discipline alternative. Elle manque de filière de formation initiale pour les instituteurs comme pour les professeurs de lycée; c'est seulement au niveau de la formation continue qu'ils peuvent acquérir un savoir-faire approprié, ce qu'ils font sur la base du volontariat. Le système en vigueur a en outre le défaut de nourrir toute une série d'oppositions: opposition entre les confessions catholique et protestante ; opposition entre celles-ci et les confessions orthodoxe, islamique, judaïque, etc. ; opposition enfin entre croyants et laïcs, car dans cette optique, l'éthique est considérée comme l'antipode de la religion, bien que toute l'histoire de la philosophie soit là pour prouver l'absurdité d'une telle conception.

Or, aux côtés de Brême et de Berlin, le Brandebourg refuse d'adhérer au système dominant. Ce Land se flatte en effet d'une tradition de tolérance qui en avait fait un refuge pour les huguenots, les Tchèques et les Autrichiens réformés, et qui a fait dire au roi Frédéric II que chacun devait pouvoir atteindre le salut à sa façon. Aussi bien la L.E.R. viset-elle à surmonter les oppositions que nous avons mentionnées. Mais les dirigeants de l'Église protestante ne cessent d'invoquer l'article 7 de la Loi fondamentale fédérale, tandis que le gouvernement du Brandebourg doit se réclamer de l'article 141 (la clause brêmoise) pour soutenir sa position. En dernier ressort, le problème est d'ordre financier. L'Église a commencé par exiger que le Land lui paye $90 \%$ du coût d'un enseignement confessionnel qu'elle a réussi à intercaler dans le tissu de la L.E.R. Elle veut maintenant imposer le schéma fédéral, aux dépens de la caisse régionale. Comme on le voit, la question met aussi en jeu des rapports de force idéologiques.

Les élections de 1994 ont renforcé les positions des sociaux-démocrates au Brandebourg. Le gouvernement régional est aujourd'hui entre leurs mains. À partir de 1995, la L.E.R. devra s'enseigner dans l'ensemble du Land, avec le statut de discipline obligatoire. Mais il $y$ aura en outre un enseignement confessionnel et un enseignement libre-penseur (comme c'est déjà le cas à Berlin), avec un statut facultatif.

Une conception originale est-elle condamnée à disparaître ?

\section{RÉSUMÉS}

Présentation d'une expérience-modèle réalisée dans le Land du Brandebourg au début des années quatre-vingt-dix. Issue des projets de réforme conçus durant la flambée démocratique de 1989, mobilisant des enseignants et des élèves volontaires, cette expérience prélude à l'institution d'un enseignement éthique dans l'ensemble des écoles du Brandebourg. Sa généralisation se heurte cependant à des difficultés juridiques qui découlent des particularités de l'histoire allemande dans le domaine de l'instruction confessionnelle et éthique. 
INDEX

Index géographique : Allemagne, Europe

Mots-clés : éducation morale, éducation religieuse, éthique, réforme de l'enseignement, système éducatif, valeur

\section{AUTEURS}

\section{JOACHIM WILKE}

Docteur en philosophie, chercheur, Akademia der Wissenschaften, Allemagne

\section{URSULA WILKE}

Docteur en éthique, Allemagne 